\title{
NOVAS TECNOLOGIAS APLICÁVEIS NO ENSINO DO DESENHO TÉCNICO: POSSIBILIDADES DE OTIMIZAÇÃO DO APRENDIZADO NOS CURSOS DE DESIGN DE PRODUTO
}

Bárbara Arantes de Paula

Universidade do Estado de Minas Gerais

barbra.arantes@gmail.com

Carlos Alberto Silva de Miranda

Universidade do Estado de Minas Gerais

carlosasmiranda@gmail.com

Resumo: O desafio que se apresenta ao ensino de Desenho Técnico, é um cenário mundial que demanda uso intensivo da ciência e tecnologia e que exige profissionais altamente qualificados. O próprio conceito de qualificação profissional vem se alterando, com a presença cada vez maior de componentes associadas às capacidades de coordenar informações, interagir com pessoas e interpretar de maneira dinâmica a realidade. As Instituições de Ensino no Brasil têm procurado, através de reformas periódicas em seus planos de ensino e conteúdos programáticos, equacionar estes problemas e solucioná-los de maneira adequada, inserindo estes conteúdos gradativamente em suas grades. Neste trabalho, descrevemos a importância do desenho técnico para o profissional de design, descrevendo as aptidões e habilidades necessárias para o desenvolvimento desta atividade, que faz parte do processo projetual, bem como descrevemos sucintamente os principais processos de visualização aplicáveis. Estes disponibilizados a partir da oferta de tecnologia proveniente de diversos meios, dentre os quais, citamos os principais que vem sendo investigados. Concluímos, ressaltando a importância do desenvolvimento de pesquisas que norteiem processos de seleção e avaliação da aplicabilidade destas novas tecnologias.

Palavras-chave: Design de produtos, desenho técnico, ensino, novas tecnologias de visualização

\begin{abstract}
The challenge facing the teaching of Technical Drawing, is a global setting that requires intensive use of science and technology and that requires highly qualified professionals. The concept of professional qualification has been changing with the increasing presence of components associated with the ability to coordinate information, interact with people and interpret dynamically reality. The education institutions in Brazil have sought through periodic reforms in their educational plans and program content, solve these problems and solve them properly, inserting these contents gradually in their grids. In this paper, we describe the
\end{abstract}


importance of the technical design for the professional design, describing the skills and abilities necessary for the development of this activity, which is part of the design process, and briefly describe the main applicable process visualization. These available from the technology supply from various means, among which we mention the key that is being investigated. We conclude by emphasizing the importance of developing research to guide the selection process and evaluation of the application of these new technologies.

Keywords: Product design, technical drawing, teaching, new display technologies

\section{INTRODUÇÃO}

O desenho é considerado, desde os primórdios da humanidade, como uma das formas mais eficazes de comunicação entre os seres humanos, além de ser um dos principais métodos de registro de pensamentos e ideias desde as civilizações mais primitivas. Atualmente se tornou possível, através dos registros encontrados em forma de desenho, reconstruir e compreender a história dos povos antigos quando nos apropriamos dos registros deixados por eles.

Existem inúmeras formas de registrar tecnicamente um objeto, haja visto que as habilidades de desenho evoluíram em conjunto com o modo de vida humano e os artefatos tecnológicos que nos cerca. Para registros técnicos de métodos de produção, modelos arquitetônicos ou peças mecânicas são utilizadas, mais comumente, ferramentas do desenho técnico de forma a se padronizar de forma normativa o método de comunicação.

O desenho técnico, que também pode ser denominado de representação técnica ou detalhamento técnico, é um ramo especializado do desenho caracterizado por sua estrutural normalização e pela apropriação que faz dos conteúdos da geometria descritiva e analítica. Embora brevemente descrita, essa área de conhecimento exige complexos referenciais teóricos, além da habilidade de visualização espacial, para ser assimilada, uma vez que faz parte da grade curricular de diversos cursos superiores e técnicos nas áreas de design, arquitetura, engenharias e demais cursos diretamente relacionados à produção industrial.

Com a evolução da tecnologia, a disciplina de desenho técnico pôde contar com a intensa colaboração de softwares para suportar seu desenvolvimento, seja no próprio desenho ou na execução das tarefas relacionadas. Porém, independente de sua evolução, é consenso entre grande parte dos acadêmicos, a necessidade do aprendizado tradicional, que utiliza os materiais de desenho praticado à mão. Por mais que a utilização de softwares de desenho técnico traga mais rapidez à disciplina e o aproxime aos processos de produção modernos, em especial os automatizados, a necessidade de manipulação correta das ferramentas digitais necessita do aprendizado inicial do desenho que, quando aprendido à mão, é desenvolvido e fixado semelhantemente ao processo de alfabetização.

As disciplinas de desenho técnico são comumente conhecidas por seu denso conteúdo normativo, acompanhadas de um rigor técnico pleno, porém extremamente 
necessário à atividade. Este rigor, por ser normatizado, permite que esta ferramenta se posicione como eficiente mecanismo de comunicação, onde a linguagem é única e universal, extrapolando as fronteiras da liguagem falada e/ou escrita. Desta forma, as aulas desta disciplina, assim como os materiais didáticos referentes a ela, permanecem os mesmos, a pelo menos 30 anos. Este fato caracteriza a problemática, que reside no fato que os processos industriais, assim como a formação cognitiva e acadêmica dos alunos, mudou de forma brusca, se modernizando ao longo dos tempos, apresentando não só novos problemas, como novas facilidades e distintas necessidades no processo de aprendizagem. Disto surge a necessidade de se desenvolver pontes de conhecimento e aplicabilidade no cotidiano, afugentando aquela ideia de memorizar o que foi dito e repeti-lo assim que necessário, conforme reflete Sforni (2004, p. 64):

\footnotetext{
"Apesar do percurso descrito (lógica formal) ser uma importante forma de conhecimento racional, é insuficiente para a interação plena do indivíduo com o conhecimento na época atual. O desenvolvimento científico e tecnológico, a especialização do saber e a complexidade da linguagem científica, marcada pela matematização e formalização do conhecimento, tornam a relação do homem com seu próprio meio social cada vez mais mediada por conceitos, códigos e signos que não são diretamente assimilados pela percepção ou raciocínio lineares. Ser sujeito da própria história é tarefa cada vez mais árdua às pessoas comuns que se sentem incapazes de interagir com a ciência - esse "mundo estranho" que não lhes pertence. Se a ciência estivesse sempre a serviço do homem, a preocupação com essa situação talvez não fosse tão importante"
}

Isto posto, é percebido que adentramos um cenário acadêmico fluido, onde o professor não é mais o emanador de conhecimento, mas sim um articulador de ideias e condutor do desenvolvimento. Desta forma, essa experiência didática se apropriou de uma metodologia de design, o metaprojeto, que se esmera em atingir não somente o requisito central do progresso de uma atividade: se no design o requisito básico é o efetivo desenvolvimento de um produto ou serviço, no processo educacional o requisito é adquirir o conhecimento estrutural do assunto lecionado. Todavia, podemos dizer que as duas áreas foram drasticamente afetadas pelo processo de globalização e que seus demais requisitos, antes secundários, foram articulados e hoje fazem parte das expectativas elementares da atividade: se no design, a estética, a interface, a afetividade e a usabilidade são também conhecidos como fatores determinantes de qualidade (MORAES, 2010, p. 20), quando se trata do processo educacional e assimilação de conteúdos, saber todas as regras estabelecidas pela ABNT não é mais a principal finalidade da disciplina. No entanto, o aluno tem que se formar habilitado para ler e assimilar novas regras constantemente, dominando sua aplicabilidade, tal qual a necessidade de sua utilização, otimizando os métodos de produção, documentação e todos os aspectos produtivos aos quais se relaciona.

\section{A IMPORTÂNCIA DO DESENHO PARA O PROFISSIONAL DE DESIGN}

Em sua atuação profissional, o designer se utiliza do desenho como forma de comunicação, pela qual são exteriorizados os pensamentos e categorizados os problemas, sendo esta uma linguagem também de persuasão para comercializar aquilo que é ideia a um determinado cliente. Além de ser um meio estruturalmente técnico para comunicar informações necessárias e precisas àqueles que fabricam, montam e comercializam um produto. Desenho é, então, uma ferramenta para a transmissão de 
informações em diversos estágios do desenvolvimento de um produto. Seja o desenho conceitual, que se encarrega de demonstrar as características principais da estruturação de um projeto; no desenho de apresentação, que especifica o aspecto final proposto, prestes à materialização do produto; ou no desenho técnico, método responsável por detalhar a complexidade das formas e estruturas tridimensionais dos artefatos, assim como os métodos de fabricação, materiais e possíveis processos, gerando a documentação oficial do projeto.

A principal incumbência desta disciplina se dá em habilitar os futuros bacharéis em design a ler, compreender e desenvolver detalhamentos técnicos de produtos de acordo com as normas estabelecidas pela Associação Brasileira de Normas Técnicas (ABNT), em conformidade com a normatização mundial gerida pela Organização Internacional para Padronização (ISO). Desta forma, o aluno qualificado na disciplina é considerado hábil a se comunicar com os demais profissionais que também detenham conhecimento prévio desse método de comunicação, independente das áreas de atuação, propiciando o pleno desenvolvimento projetual em conjunto com os demais profissionais.

$\mathrm{O}$ ato de desenhar caracteriza um diálogo entre o designer e o papel, durante seu processo de criação. Uma vez que o profissional não detém a habilidade do desenho, ou a possui com debilidades, este estará limitado em seu processo de criação, visto que só consegue projetar aquilo que põe efetivamente no papel, explicitando os métodos, processos, raciocínios e toda a complexidade da forma que pretende desenvolver, decodificada em traços. As habilidades de desenho são comumente atreladas ao traço do profissional, mas também são frutos da biblioteca visual que este possui: quanto mais formas o designer reconhece e exercita, melhor consegue representá-las. Esta habilidade é conhecida por percepção visual, que se traduz pela capacidade de visualizar formas, imaginar e rotacionar artefatos mentalmente assim como, ao visualizar objetos planificados, ser capaz de montá-los em tridimensionalidade, ainda que mentalmente.

A aptidão do desenho por vezes é classificada como dom, uma espécie de talento divino, como uma forma de minimizar a habilidade de uns em detrimento de outros. É verdade que alguns indivíduos possuem mais condicionamento em aprender a representar formas, todavia esta perícia se faz presente na raça humana desde os primórdios da humanidade, tendo sido também um método de comunicação propriamente eficaz, nos conduzindo ao pressuposto que a maioria das pessoas possui a mesma habilidade, variando somente a quantidade de exercícios que cada um realiza em função de aprimorar este know-how. Desenhar deveria ser parte estrutural no processo educacional do indivíduo, complementar à formação integral dos cidadãos, não somente aos que desenvolvem tais funções profissionalmente, ampliando as capacidades de comunicação e expressão de cada ser.

\section{HABILIDADES E VISÃO ESPACIAL}

Segundo Montenegro (2003), as habilidades são o resultado de múltiplas combinações de idéias, pensamentos, dados, conhecimentos prévios e tarefas que um indivíduo é capaz de fazer e das informações úteis que este indivíduo obteve a partir da aplicação de uma ou mais dessas habilidades. A habilidade ou inteligência espacial envolve pensar em imagens, bem como a capacidade de perceber, transformar e recriar diferentes aspectos do mundo visual e espacial. Indivíduos com alta habilidade 
espacial possuem sensibilidade apurada para identificar detalhes visuais, esboçam facilmente idéias de forma gráfica e facilmente se orientam no espaço tridimensional.

Esse contexto encontra-se aplicado em diversas áreas profissionais, que usam frequentemente as habilidades espaciais, podendo aqui se destacar os profissionais de design.

De acordo com Choi (2001), as habilidades espaciais podem ser descritas através de três categorias distintas: a rotação mental, a percepção espacial e a visualização espacial. A rotação mental é a habilidade de manipular, rotacionar, torcer ou inverter objetos tridimensionais de forma imaginativa, no momento da compreensão. $\mathrm{O}$ indivíduo com essa competência deve ser capaz de visualizar e rotacionar mentalmente os objetos em posições diferentes. A percepção espacial refere-se à habilidade de determinar relacionamentos espaciais a partir de informações visuais, o que coincide com o senso cinestésico apurado. A visualização espacial consiste na manipulação de problemas visuais complexos imaginando os movimentos relativos das partes internas de uma imagem, compondo assim a solução.

Em adição às categorias apresentadas por este autor, Maier (1994) apud Kaufmann \& Schmastieg (2003) destaca ainda as relações espaciais e a orientação espacial. As relações espaciais se referem às relações que podem ser estabelecidas através de elementos dispostos no ambiente, podendo-se utilizar pontos de referência. Já a orientação espacial, se refere à habilidade de se orientar no espaço à medida que objetos ou eventos são apresentados (SHELTON \& McNAMARA, 2001).

As disciplinas de Desenho Técnico tem como objetivo principal capacitar o aluno a se comunicar através de representações gráficas, mas constituem-se num valioso instrumento de trabalho para o desenvolvimento da visualização espacial (GITTLER \& GLÜCK, 1998). Essas disciplinas são fundamentais no processo de formação dos profissionais de design, tendo em vista que parte fundamental do trabalho dos mesmos consiste na concepção de objetos através do desenvolvimento de projetos e planejamento de processos de produção. Para um melhor desempenho, sob este aspecto, é necessário que a capacidade de visualização espacial seja desenvolvida, habilidade esta nem sempre satisfatória em alguns alunos ingressantes (VELASCO \& KAWANO, 2002).

Diversos testes podem ser empregados para avaliar o nível de habilidade de visualização espacial de um indivíduo (SEABRA, 2004; GORSKA \& JUSCAKOVA, 2003; RIZZO, et al., 2000; TSUTSUMI, 1999; ASCHER, 2000; GUAY, 1977; LEHMANN, 2000; QUAISER-POHL, 2003; SHEPARD \& METZLER, 1971; VANDENBERG \& KUSE, 1978; STRONG \& SMITH, 2001).

O MRT - Mental Rotation Test, ou teste de rotação mental, (ASCHER, 2000; LEHMANN, 2000; SHEPARD \& METZLER, 1971; VANDENBERG \& KUSE, 1978) foi desenvolvido para determinar-se o tempo necessário que um indivíduo necessita para reconhecer se duas figuras são iguais ou não. O objetivo é encontrar entre as alternativas, as duas que representem a figura de referência. Sua medida é correlacionada com a habilidade de rotação mental (CAISSIE; VIGNEAU \& BORS, 2009). A Figura 1 mostra um exemplo dentre as 20 questões do MRT: 
1.
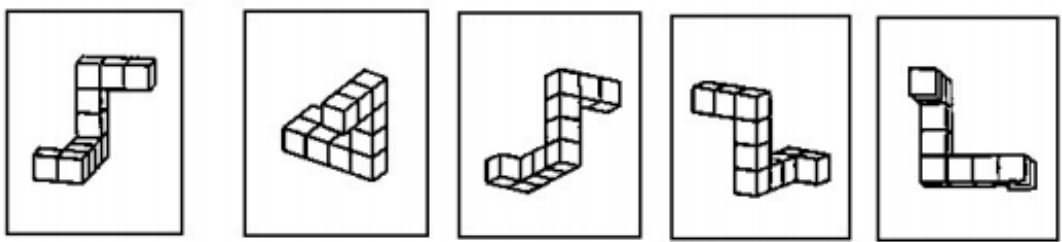

Figura 1: Exemplo de questão do MRT - Mental Rotation Test.

Fonte: CAISSIE; VIGNEAU \& BORS (2009)

O MCT - Mental Cutting Test, ou teste de corte mental, possui 25 questões, onde cada uma apresenta uma perspectiva de um objeto sendo cortado por um plano. O objetivo é assinalar, dentre as cinco opções apresentadas, a que corresponde à forma do perímetro da seção resultante do corte do objeto pelo plano dado. A Figura 2 ilustra um exemplo de questão do MCT (TSUTSUMI, 2004; VELASCO \& KAWANO, 2002):
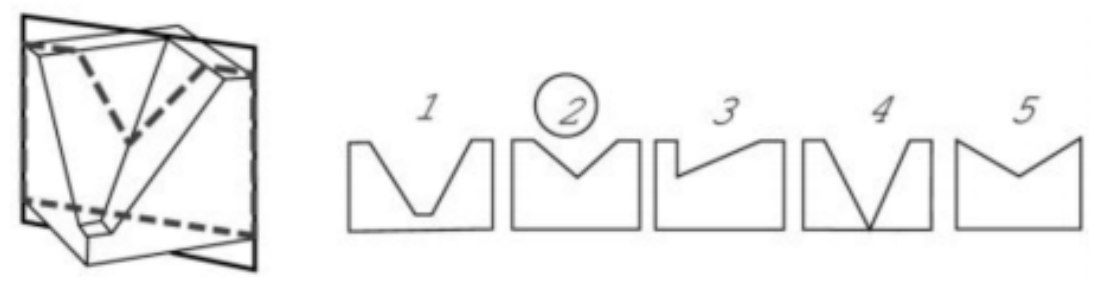

Figura 2: Exemplo de questão de MCT.

Fonte: TSUTSUMI, 2004.

O TVZ - Teste de visualização (Test de Visualización) possui 32 questões envolvendo o desdobramento de um cubo. O objetivo é identificar qual letra e em qual posição ela aparece na face solicitada do cubo desdobrado. A Figura 3 mostra um exemplo de questão do TVZ (VELASCO \& KAWANO, 2002):
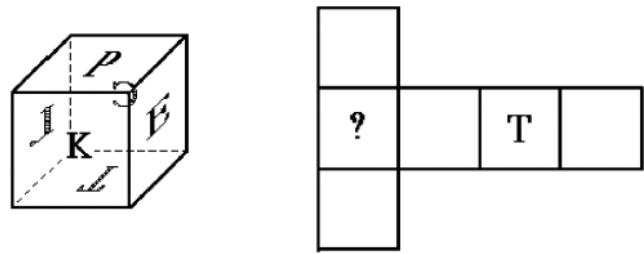

Figura 3: Exemplo de questão do TVZ.

Fonte: ADÁNEZ \& VELASCO(2002).

O VR - MRT apresentado por Rizzo, et al. (2000), consiste na aplicação do teste MRT - Mental Rotation Test em um ambiente virtual tridimensional estereoscópico.

O MCT - Schnitte, apresentado por Quaiser-Pohl (2003), é um teste que não pode ser resolvido olhando-se para as figuras apresentadas, dependendo exclusivamente da imagem mental.

O SMCT (TSUTSUMI, 1999), consiste na aplicação do teste MCT - Mental Cutting Test, porém apresentando sólidos em ambiente estereoscópico.

\section{NOVAS TECNOLOGIAS APLICADAS NO ENSINO DO DESENHO TÉCNICO}

As novas Tecnologias de Informação e Comunicação vêm se tornando cada vez mais presentes no cotidiano das pessoas e mais presentes ainda nas escolas e universidades. Os sistemas computacionais também estão presentes em grande parte 
dos estabelecimentos. Neste cenário, o planejamento da educação não poderia ser diferente. Além dos computadores interligados em rede e com acesso à internet, disponibilizando recursos para um número infinito de possibilidades de pesquisa e também com sistemas que atendem determinadas situações e disciplinas específicas. (BERGAMASCHIA \& SILVEIRA, 2012). As múltiplas oportunidades de pesquisa em visualização espacial emergiram em consequencia das novas tecnologias. A visualização estereoscópica, por exemplo, talvez possa auxiliar o processo de ensinoaprendizagem facilitando a visualização e compreensão de situações espaciais complexas e, por conseguinte, apoiando o desenvolvimento da cognição espacial do estudante, especialmente daqueles com menor habilidade (SEABRA \& SANTOS, 2004).

A grande evolução da informática e da eletrônica trazem a oportunidade de empregar novos recursos tecnológicos para o ensino/aprendizagem de disciplinas na área gráfica. Com o advento de novas tecnologias (como por exemplo os óculos de realidade virtual que fazem uso de celulares do tipo smartphone, onde as imagens são projetadas com focos distintos entre os dois olhos, gerando uma imagem tridimensionalizada - Figura 4), pode-se agora utilizar com mais facilidade e sucesso, técnicas estereoscópicas, realidade virtual, ou mesmo realidade aumentada (augmented reality) no aprendizado de Geometria e Desenho Técnico. A finalidade básica de seu uso é sempre o de facilitar a visualização espacial.

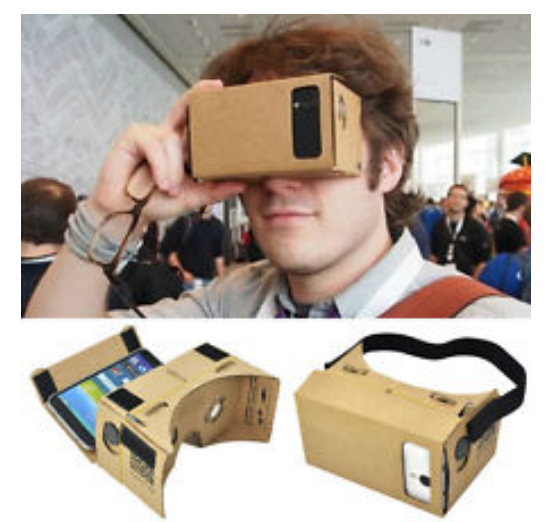

Figura 4: Óculos Google Cardboard RV - em papelão ondulado, aplicado em projeções estereoscópicas, fazendo-se uso de celulares do tipo smartphone.

Fonte: Google Imagens.

A prototipagem rápida é uma tecnologia que permite a geração de protótipos automaticamente a partir de modelos 3D desenvolvidos em CAD. Os processos de fabricação rápida de protótipos de peças são bastante variados, classificando-se em subtrativos, aditivos e compressivos. Os mais comuns são os processos subtrativos, onde um bloco de material é esculpido para gerar a forma projetada. Atualmente existem no mercado equipamentos CNC (Comando Numérico Computadorizado) bastante pequenos e de custo reduzido, que permitem o uso de técnicas de prototipagem rápida no ensino de Desenho Técnico (Figura 05). 


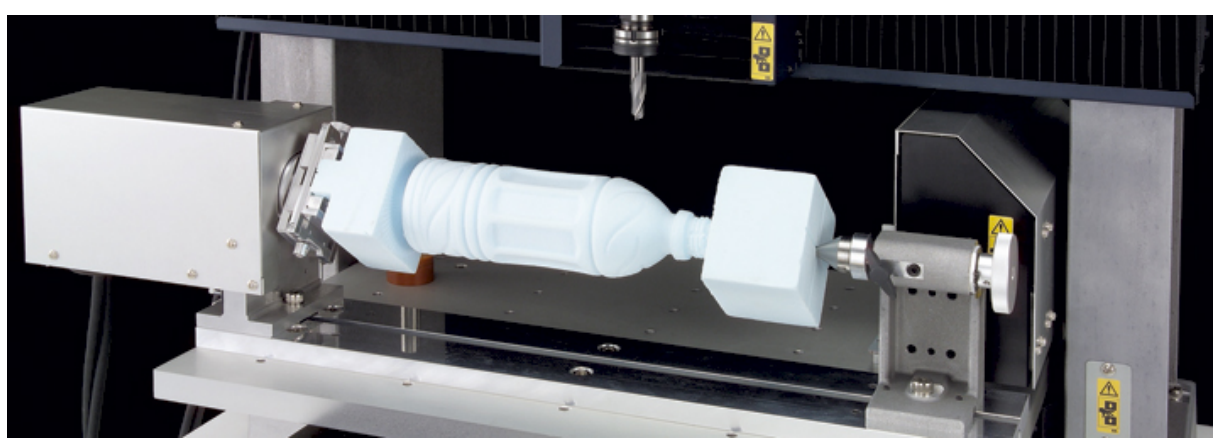

Figura 5: Exemplo de máquina de prototipagem rápida por subtração, com tecnologia CNC Fonte: Roland (www. rolanddga.com)

Os processos compressivos comprimem um material semi-sólido ou líquido até a forma desejada. Os mais modernos são os processos aditivos tais como os fotopolímeros (resina líquida solidificada por luz com comprimentos específicos). Ou o mais utilizado atualmente, por sua acessibilidade e baixo custo de instalação: o processo de prototipagem 3D. O processo Fused Deposition Modeling (FDM) ou "Deposição de Material Fundido" é o processo atualmente mais acessível em termos de custos de equipamento e de matéria-prima, no qual um determinado material em estado plástico, é seletivamente depositado em camadas, em uma plataforma através de um bico extrusor, conforme Figura 6, a partir de um modelo paramétrico que pode ser desenvolvido até mesmo a partir de um aplicativo de celular. Para completar cada camada, este bico vai depositando o material em todo o contorno da seção e, depois de terminado, passa a preencher o conteúdo do contorno, geralmente em movimentos de vai e vem. Depois que a primeira camada é terminada, o bico sobe alguns décimos de milímetro e inicia a fabricação da segunda camada, e assim vai fazendo sucessivamente até que a peça esteja completa.
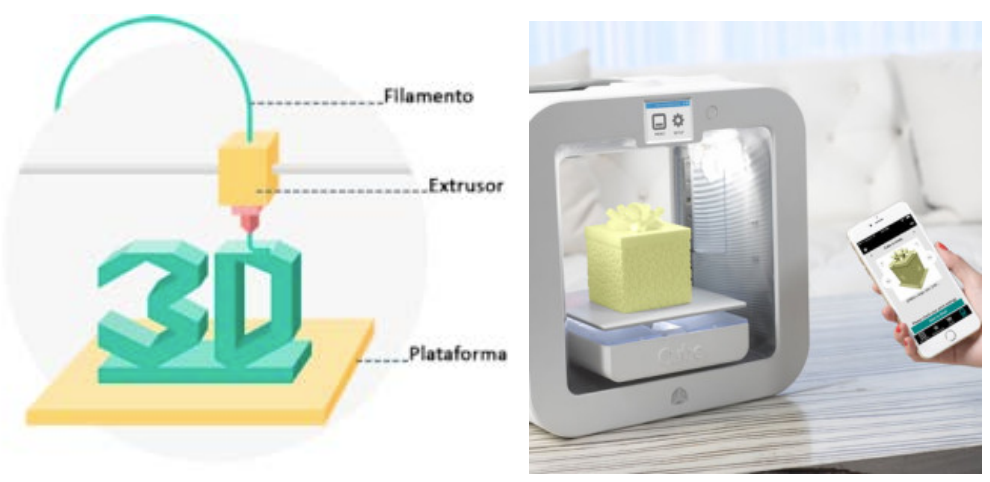

Figura 6: Esquema ilustrativo da impressão 3D através do processo FDM e impressora 3D Fonte: Adaptado de THRE3D, 2014

A utilidade de tais equipamentos como apoio ao ensino de Desenho pode se dar tanto através do uso dos modelos físicos facilmente gerados por eles como também através da atividade de programação de tais máquinas pelos próprios alunos. O uso de modelos físicos pode ocorrer nos primeiros contatos do aluno com o desenho, pois dispensa totalmente a visualização espacial, sendo a maneira mais intuitiva de representação de uma forma tridimensional. Neste processo, dois sentidos (visão e tato) estão envolvidos, facilitando a compreensão da forma. Em seguida, na prática de esboço, a manipulação do modelo físico também é interessante pois facilita 
a memorização das formas e porque permite a orientação do objeto em inúmeras posições, enriquecendo o exercício (MAFALDA \& KAWANO, 1999). Os modelos físicos ainda podem ser úteis na aquisição do conceito de formação das vistas ortográficas pela projeção na "caixa de vidro". As várias vistas ortográficas podem ainda ser rapidamente visualizadas (aproximadamente) através das várias faces do objeto. Por fim, na axonometria ortogonal, as várias perspectivas podem ser ensaiadas pelo aluno alterando a posição do modelo em suas mãos. Ou mesmo a aplicação de ambos: o modelo físico, em madeira, isopor, resina (obtidos, por exemplo, através da prototipagem subtrativa, em máquinas $\mathrm{CNC}$ ), ou mesmo impressos em prototipagem 3D, aliados à projeção tridimensional de modelos paramétricos executados em sistemas CAD (Figura 7).

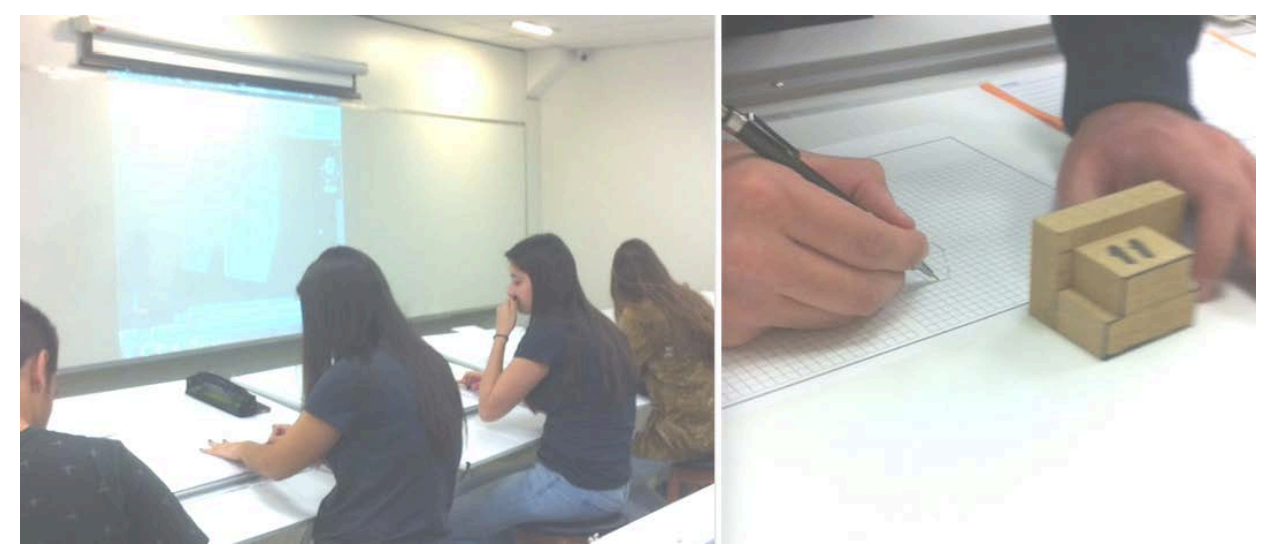

Figura 7: Uso de modelos físicos em madeira, aliados à projeção de modelos em 3D através de modelagem em CAD aplicados no didática do ensino de Desenho Técnico.

Fonte: Elaborado pelo autor, com base na pesquisa realizada.

Portanto, as diferentes manifestações dos softwares, desde os sistemas CAD introduzidos nos anos 1980 e 1990, passando pelos programas de Geometria Dinâmica, até as mais novas aplicações na Internet, são todas oportunidades para modernizar o ensino gráfico, incrementar o aprendizado e motivar o estudante. As novas tecnologias incorporadas no hardware e no software modernos abrem inúmeras possibilidades para o ensino de Desenho e Geometria.

Por fim, a motivação despertada nos alunos pelo uso destes interessantes equipamentos também não deve ser desprezada, já que normalmente se tratam de estudantes dos períodos iniciais dos cursos de Design, os quais tradicionalmente apresentam elevadas taxas de evasão. As disciplinas de Desenho Técnico apresentam uma forte ligação, pela habilidade inicial exigida, com o fator aptidão. Porém, isso pode ser contornado, ou amenizado, com a aplicação dessas tecnologias.

\section{CONCLUSÃO}

O desafio que se apresenta ao ensino de Desenho Técnico, é um cenário mundial que demanda uso intensivo da ciência e tecnologia e que exige profissionais altamente qualificados. O próprio conceito de qualificação profissional vem se alterando, com a presença cada vez maior de componentes associadas às capacidades de coordenar informações, interagir com pessoas e interpretar de maneira dinâmica a 
realidade. As Instituições de Ensino no Brasil têm procurado, através de reformas periódicas em seus planos de ensino e conteúdos programáticos, equacionar estes problemas e solucioná-los de maneira adequada, inserindo estes conteúdos gradativamente em suas grades.

Faz-se necessário, portanto, que se proponham novas estratégias de ensino em Desenho Técnico, com a utilização de recursos computacionais, inserindo nas práticas didáticas a possibilidade de visualização dos objetos em três dimensões, através da apropriação e do uso dessas novas tecnologias de reprsentação.

Através da visualização dos objetos de geometria espacial em 3D, o aluno terá em tese, uma maior percepção do objeto, auxiliando então os procedimentos de ensino e aprendizado do desenho técnico. A busca de soluções que trazem ao profissional de design a melhoria das suas condições de trabalho, bem como de aprendizado, na otimização dos recursos didáticos empregados no ensino de suas disciplinas básicas, aqui destacando as disciplinas de representação técnica.

A origem das tentativas de aplicação de tecnologias de computação gráfica, realidade virtual e Internet nas disciplinas de desenho técnico, remonta ao início dos anos 1990, conforme já falado. Nessa época, pesquisas identificaram e caracterizaram as dificuldades de visualização dos alunos quando confrontados com objetos reais por um lado e, por outro, com as suas representações em projeções ortogonais (PRATINI \& FALEIRO, 2001). Passada uma experiência bem-sucedida de ensino com aplicação de modelagem tridimensional, estes autores desenvolveram uma tentativa de ensino com auxílio de programas CAD. Nesta, verificaram que os alunos envolviam-se mais com o programa computacional e com os seus comandos do que com a visualização dos objetos desenhados ou com a técnica, as normas e a prática do Desenho Técnico. Portanto, o emprego dessas novas tecnologias merece atenção e estudos, de forma a se promover o seu uso e aplicação de forma eficiente, na otimização do ensino e aprendizagem. É mister o desenredo de pesquisas que norteiem processos de seleção e avaliação da aplicabilidade destas novas tecnologias, que são de domínio e familiaridade por parte da geração de discentes com os quais temos nos deparado, em sala de aula.

\section{REFERÊNCIAS}

ADÁNEZ, Gerardo Prieto; VELASCO, Angela Dias. Construção de um teste de visualização a partir da psicologia cognitiva. Avaliação Psicologica, v. 1, n. 1, p. 39-47, 2002.

ASCHER, E. A. Mental Rotations in Artists and Non-Artists. In: Journal of the Sciences, 2000.

CAISSIE, André F.; VIGNEAU, François; BORS, Douglas A. What does the Mental Rotation Test measure? An analysis of item difficulty and item characteristics. Open Psychology Journal, v. 2, n. 1, p. 94-102, 2009.

CHOI, J. Sex Differences in Spatial Abilities in Humans: Two Levels of Explanation. In: VOKEY, J. R.; ALLEN, S. W. Psychological Sketches, Department of Psychology and Neuroscience, University of Lethbridge, 5a ed., 2001. 
GITTLER, G. \& GLÜCK, J. Differential Transfer of Learning: Effects of Instruction in Descriptive Geometry on Spatial Test Performance. In: Journal of Geometry and Graphics, v. 2, no 1, pp. 71-84, 1998.

GORSKA, R. A. \& JUSCAKOVA, Z. A Pilot Study of a New Testing Method for Spatial Abilities Evaluation. In: Journal for Geometry and Graphics, v. 7, no 2, pp. 237-246, 2003.

GUAY, R. B. Purdue Spatial Visualization Test: Rotations. West Lafayette: Purdue Research Foundation, 1977.

KAUFMANN, H \& SCHMALSTIEG, D. Mathematics and Geometry Education with Collaborative Augmented Reality. In: Computers \& Graphics, v. 27, pp. 339- 345, 2003.

LEHMANN, W. Group Differences in Mental Rotation. Institut fur Psychologie, Ottovon-Guericke-Universitat Magdeburg, 2000.

MAFALDA, R.; KAWANO, A. Aplicações de Comando Numérico na Disciplina Desenho para Engenharia. Boletim Técnico PCC/EPUSP, 2000.

MAIER, P. H. Räumliches Vorstellungsvermögen. Lang, Frankfurt a.M., Berlim, Bern, New York, Paris, Wien 1994.

MONTENEGRO, G. Habilidades Espaciais: exercícios para o despertar de idéias. Santa Maria: SCHDs, 2003.

MORAES, D. Metaprojeto: o design do design. São Paulo: Edgard Blücher, 2010.

BERGAMASCHIA, M. \& SILVEIRA, I. F. O uso de Realidade Aumentada como apoio ao ensino de Desenho Técnico para o curso de Engenharia: um Estudo de Caso. LACLO 2012 - Séptima Conferencia Latinoamericana de Objetos y Tecnologías de Aprendizaje. Vol 3, No 1, Guayaquil Ecuador, 8-12 de octubre, 2012.

PRATINI, E. \& FALEIRO, J. Uma nova metodologia de apoio à visualização no ensino de desenho técnico e geometria descritiva. Libro de Ponencias del 5 to Congreso Iberoamericano de Gráfica Digital SIGRADI-2001. Experiências pedagógicas, p.201-203. Ediciones Universidad del Bío-Bío, 2001, 500 pp.

QUAISER-POHL, C. The Mental Cutting Test "Schnitte" and The Picture Rotation Test Two New Measures to Assess Spatial Ability. In: International Journal of Testing, v. 3, no 3, pp.219-231, 2003.

RIZZO, A. A. et al. Virtual Reality Applications for the Assessment and Rehabilitation of Attention and Visuospatial Cognitive Processes: an update. In: Proceedings of the third international conference on disability, virtual reality and associated technologies, Alghero, Italy, pp. 197-207, 2000.

SANTOS, E. T. Novas tecnologias no ensino de desenho e geometria. Anais do I Encontro Regional do Vale do Paraíba de Profissionais do Ensino da Área de Expressão Gráfica, p. 71-81, out. 2000, Lorena, SP.

SEABRA, R. D \& SANTOS, E. T. Proposta de desenvolvimento da habilidade de visualização espacial através de sistemas estereocópicos. Actas del EGRAFIA $4^{\circ}$ Congreso Nacional y 1 ro. Internacional Rosario, Argentina -6 a 8 de octubre de 2004. 
SFORNI, M. S. F. Aprendizagem Conceitual e Organização do Ensino: Contribuições da Teoria da Atividade. Araraquara: JM Editora, 2004.

SHELTON, A. L.; McNAMARA, T. P. Systems of Spatial Referente in Human Memory. In: Cognitive Psychology, v. 43, pp. 274-310, 2001.

SHEPARD, R. N. \& METZLER, J. M. Mental Rotation of Three-Dimensional Objects. In: Science, v. 171, no 3972, pp. 701-703, 1971.

SILVEIRA, I. F. \& ARAÚJO JR., C. F. Tecnologia da Informação e Educação: Pesquisas e Aplicações. São Paulo: Andross, 2006.

STRONG, S. \& SMITH, R. Spatial Visualization: Fundamentals and Trends in Engineering Graphics. In: Journal of Industrial Technology, v. 18, № 1, 2001.

THRE3D - 3D Printing, Simplified., 2014. Disponivel em:<https://thre3d.com>. Acessado em 04/05/2016.

TSUTSUMI, E. A. Mental Cutting Test on Female Students Using a Stereographic System. In: Journal for Geometry and Graphics, v. 3, no 1, pp. 111-119, 1999.

TSUTSUMI, Emiko. A Mental Cutting Test using drawings of intersections. Journal for Geometry and Graphics, v. 8, n. 1, p. 117-126, 2004.

VANDENBERG, S. G. \& KUSE, A. R. Mental Rotations, a Group Test of ThreeDimensional Spatial Visualization. In: Perceptual and Motors Skills, v. 47, pp.599- 604, 1978.

VELASCO, A. D. \& KAWANO, A. Avaliação da Aptidão Espacial em Estudantes de Engenharia como Instrumento de Diagnóstico do Desempenho em Desenho Técnico. São Paulo: Departamento de Engenharia de Construção Civil, Escola Politécnica da Universidade de São Paulo, 2002. 